\title{
SPECTRALLY-QUANTIFIED CHEMICAL REACTIVITY OF OPTICAL FLUIDS AND MATERIALS IN THE GMACS SPECTROGRAPH FOR GMT
}

\author{
A Senior Scholars Thesis \\ by \\ TYLER WILLIAM BEHM

\begin{abstract}
Submitted to the Honors and Undergraduate Research
Texas A\&M University
\end{abstract} \\ in partial fulfillment of the requirements for the designation as \\ UNDERGRADUATE RESEARCH SCHOLAR
}

May 2012

Major: Physics

Math 


\title{
SPECTRALLY-QUANTIFIED CHEMICAL REACTIVITY OF OPTICAL FLUIDS AND MATERIALS IN THE GMACS SPECTROGRAPH FOR GMT
}

\author{
A Senior Scholars Thesis \\ by \\ TYLER WILLIAM BEHM \\ Submitted to Honors and Undergraduate Research \\ Texas A\&M University \\ in partial fulfillment of the requirements for the designation as \\ UNDERGRADUATE RESEARCH SCHOLAR
}

Approved by:

Research Advisor:

Darren DePoy

Associate Director for Honors and Undergraduate Research:

Duncan MacKenzie

May 2012

Major: Physics

Math 


\begin{abstract}
GMACS Spectrograph for GMT. (May 2012)

Tyler William Behm

Department of Physics and Astronomy

Department of Mathematics

Texas A\&M University

Research Advisor: Dr. Darren DePoy

Department of Physics and Astronomy
\end{abstract}

Spectrally-Quantified Chemical Reactivity of Optical Fluids and Materials in the

We present a preview of compatibility tests for index-matching fluids with commonly used optical assembly materials. The optical coupling fluids used in the Giant Magellan Areal Camera and Spectrograph (GMACS) for the Giant Magellan Telescope (GMT) will be selected based on these empirical results. Although we focus on fluid candidates for GMACS, the results of the conducted experiments are applicable to all instruments that use optical index-matching fluids. The experiment presented here identifies potentially corrosive matchings of fluids and materials. In the experiment, a material (RTV, polyethylene, delrin, etc.) is submerged in a quartz cuvette of fluid (Cargille liquids, glycerin, etc.). Contamination is observed by using a spectrometer to measure the absorption spectrum at various post-submersion times. The final results will track the percent degradation in the UV transmission as a function of post-submersion time. The final paper will be presented at SPIE conference 8446 . 


\section{ACKNOWLEDGMENTS}

I would like to thank Robert Barkhouser and Dr. Dan Fabricant for their suggestions on which materials and fluids to test for GMACS and spectrometers in general.

I would like to thank the National Science Foundation for its financial support of the Giant Segmented Mirror Telescope Project and consequently the support of instrumentation design for GMT.

I would like to thank Charles R. '62 and Judith G. Munnerlyn, George P. '40 and Cynthia Woods Mitchell, and their families for support of astronomical instrumentation activities in the Department of Physics and Astronomy.

Finally, I would like to especially thank my wonderful advisors, Dr. Darren DePoy, Dr. Jennifer Marshall, and Jean-Phillipe Rheault, for all their support. They took me in as an unexperienced freshman, gave me amazing guidance and opportunities throughout my college career, and helped develop most of what I am as a researcher today. None of this paper would have been possible without them. For all of these reasons, I am eternally thankful to them. 


\section{NOMENCLATURE}

$\begin{array}{ll}\text { GMT } & \text { Giant Magellan Telescope } \\ \text { GMACS } & \text { Giant Magellan Areal Camera and Spectrograph } \\ \text { RSS } & \text { Robert Stophie Spetrograph } \\ \text { DEIMOS } & \text { Deep Imaging Multi-object Spectrograph } \\ \text { RTV } & \text { Room temperature vulcanized rubber } \\ \text { UV } & \text { Ultra-violet } \\ \text { S/N } & \text { Signal-to-noise }\end{array}$




\section{TABLE OF CONTENTS}

ABSTRACT .................................................................................................ii

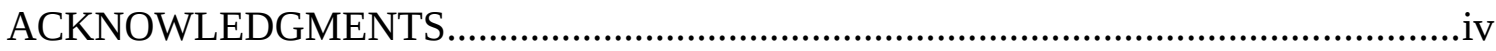

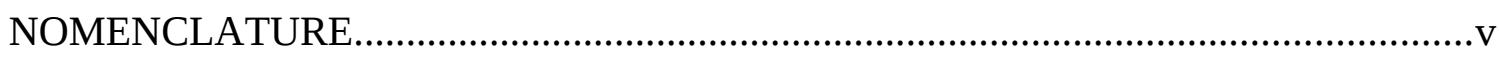

TABLE OF CONTENTS ...................................................................................vi

LIST OF FIGURES.......................................................................................... vii

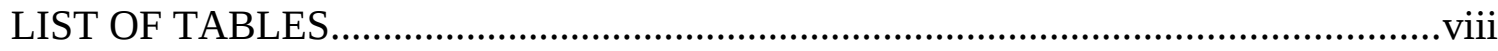

CHAPTER

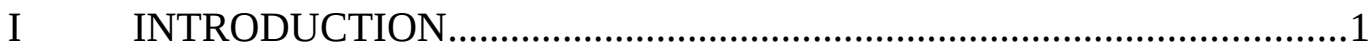

II METHOD

Setup.............................................................................. 5

Optical coupling in setup........................................................6

Monochromator grating in setup............................................

Two phase measurement............................................................8

Final setup and procedure....................................................10

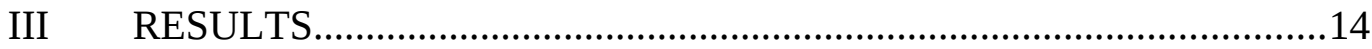

Preliminary test....................................................................... 14

Aging the contaminated samples..............................................16

IV SUMMARY AND CONCLUSIONS............................................17

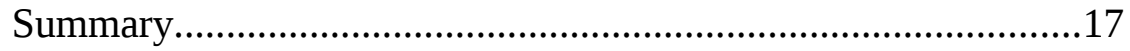

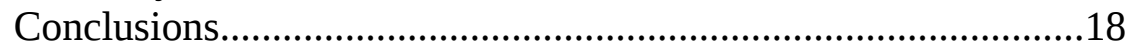

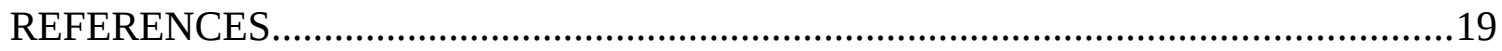

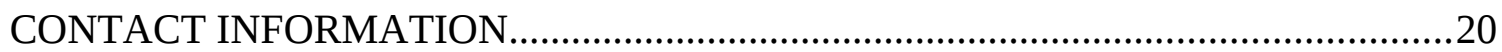




\section{LIST OF FIGURES}

FIGURE

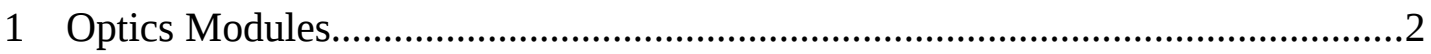

2 Indices of Refraction..................................................................................

3 UV Throughput Comparison of 5 Fibers.................................................... 7

44 Different Grating Blaze Angle's Signal-to-Noise.........................................

5 Signal-to-Noise for 2 Phase Test...................................................................

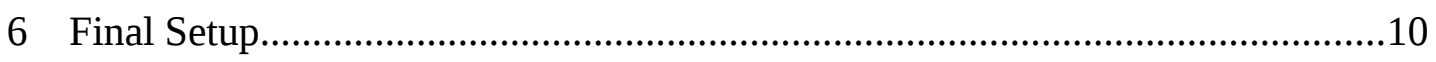

7 Glycerin w/Delrin: Transmission Curves After Contamination............................14 


\section{LIST OF TABLES}

TABLE

Page

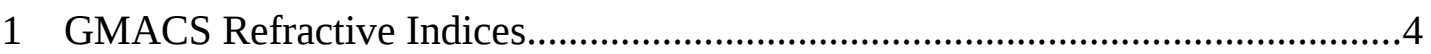

2 Fluids and Materials in Other Instruments................................................. 


\section{CHAPTER I}

\section{INTRODUCTION}

The Giant Magellan Telescope is a ground-based extremely large telescope that is currently scheduled for completion before 2020 at the Las Campanas Observatory in Chile. GMT will consist of seven $8.4 \mathrm{~m}$ (27.6 ft) diameter primary mirror segments and will have the equivalent resolving power of a $24.5 \mathrm{~m}(80.4 \mathrm{ft})$ single primary mirror and the light collecting ability of a $21.4 \mathrm{~m}$ (70.2 ft) single primary mirror (Lloyd-Hard et. al. 2006). This will give GMT over four times the light-gathering ability of Gran Telescopio Canarias which is the ground-based optical telescope with the largest effective mirror area -- same as a $10.4 \mathrm{~m}$ (34.2 ft) diameter single primary mirror -- as of the writing of this paper.

The Giant Magellan Areal Camera and Spectrograph (GMACS shown in figure 1) is a wide-field optical spectrograph for GMT. GMACS will aid GMT in its science goals of observing galaxy assembly, dark energy, first light, and reionization. GMACS is an integral part of GMT and because of this, it is imperative to ensure the integrity of the GMACS spectrograph for the decades that it will be in operation.

This thesis follows the style of Publications of the Astronomical Society of the Pacific. 


\section{Optics Modules}
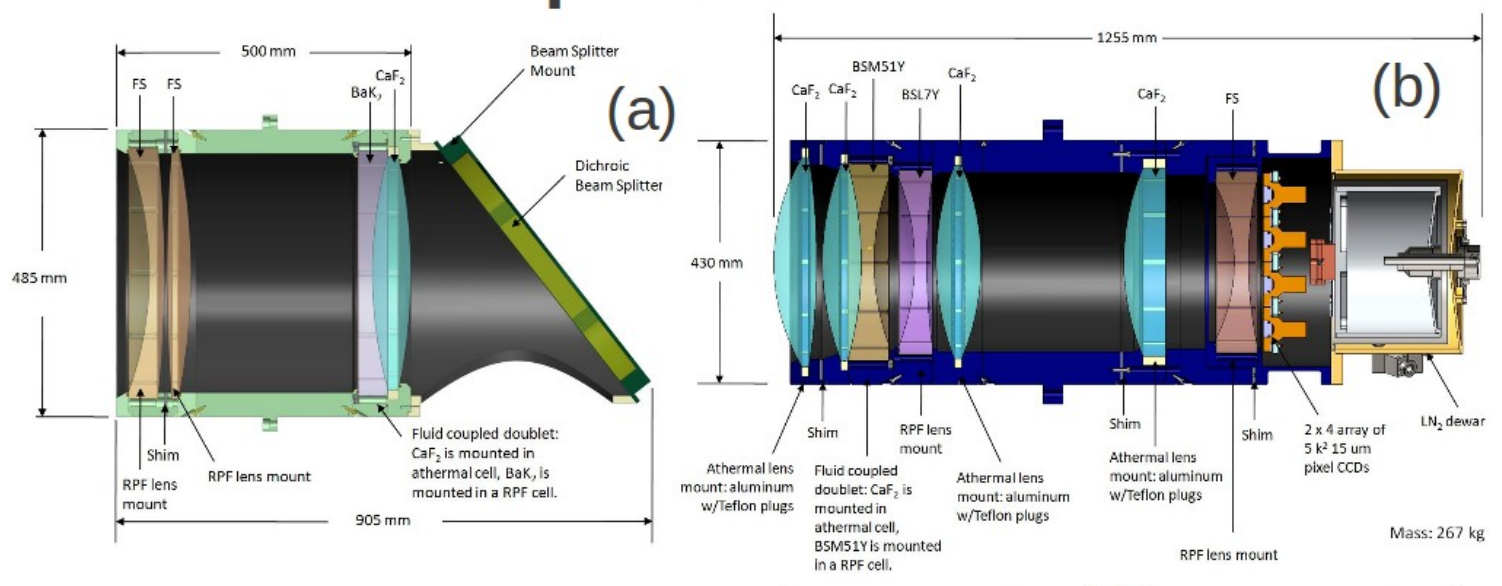

GMACS opto-mechanical assemblies:

(a) Collimator cell and structure.

(b) Blue camera showing lens arrangement, structure, and cryostat for detectors.

(c) Red camera showing various layout, structure, and design details. Also shown is the cryostat for the detectors.

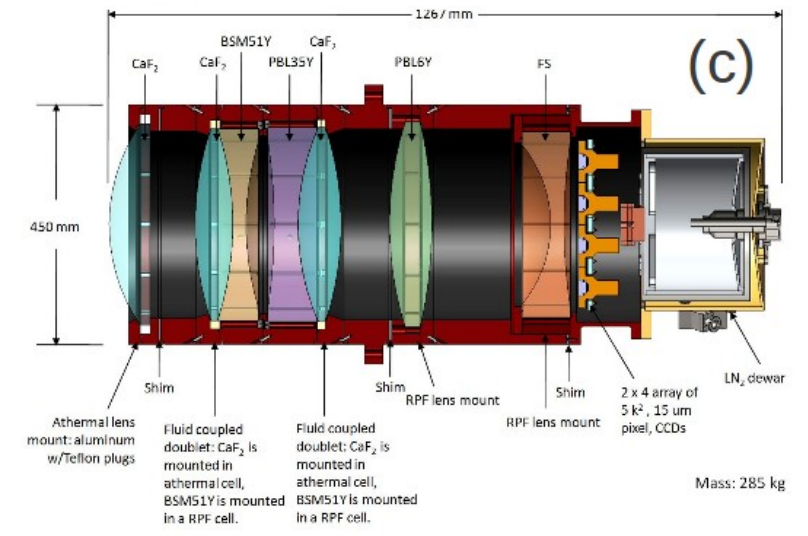

FIG. 1- Optics Modules. The GMACS spectrometer optical design contains many different lens elements. Some of the lenses consist of a convex lens and a concave lens combined together. This is called a doublet. It is very important the we fill the gap in the middle of the doublet with index matching fluids so that air does not get in and distort the optics. (Marshall et. al. 2012)

Recently, several spectrographs have been compromised by the corrosive nature of optical fluids with some materials. Two years after the commissioning of the Robert Stobie Spectrograph (RSS) on the Southern African Large Telescope, astronomers noticed a significant loss of ultra-violet transmission (Buckley et. al. 2008). With great difficulty and time lost, they diagnosed that the optical fluids had corroded the polyurethane bladder (Nordsieck, Nosan, and Schier 2010). These particles from the corrosion fell into the fluid and blocked UV light. 


\section{Indices of Refraction}

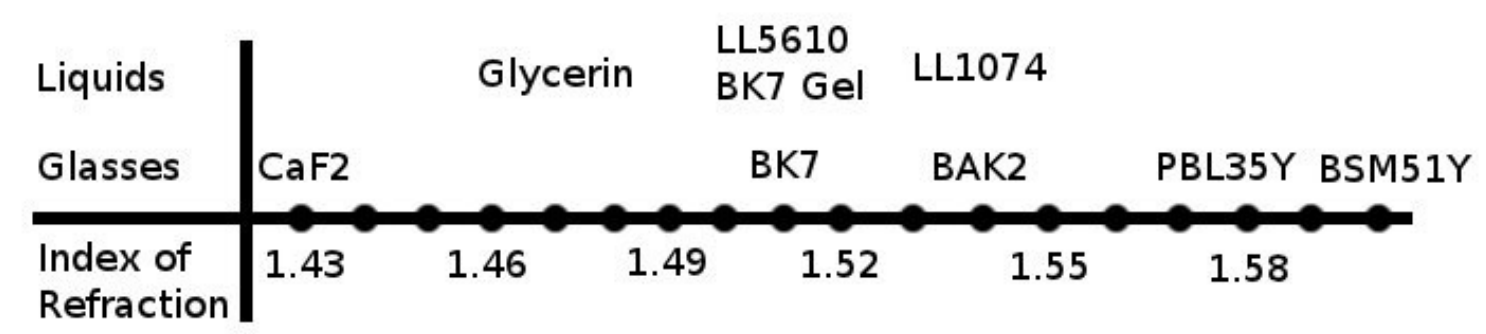

FIG. 2- Indices of Refraction. For an index-matching fluid to work as optical coupling for a lens doublet, it must have an index of refraction between those of each lens element.

Furthermore, other astronomers have taken a preemptive approach to this problem. When designing the Deep Imaging Multi-Object Spectrograph (DEIMOS), the team at UCO/Lick Observatory did a preliminary 3-month test of several optical fluid and material candidates for DEIMOS (Hilyard, Laodipis, and Faber 1999). With their research, they qualitatively identified and avoided many incompatible fluid and material pairs.

In our research, we seek to select index-matching fluids suitable for GMACS (Figure 2 and Table 1) and to conduct similar tests as those conducted by the UCO/Lick team. We want to augment these tests by measuring the absorption spectra of the fluids to obtain exact quantitative results. With our research, we hope TO ensure the integrity of the GMACS spectrograph and to provide information for future spectrograph instrumentation (Table 2). 
TABLE 1- GMACS Refractive Indices. For an indexmatching fluid to work as optical coupling for a lens doublet, it must have an index of refraction between those of each lens element.

Fluid

Glyerin

Cargille Laser Liquid Code 1074

Cargille Laser Liquid Code 5610

Cargille Optical Gel Code 081160
Index at $589.3 \mathrm{~nm}$ and $25^{\circ} \mathrm{C}$

1.4729

1.5358

1.51484

1.52

1.509

1.43385

1.60311

1.53996

1.58159

TABLE 2- Fluids and Materials in Other Instruments.

Many of the fluids and materials are popular for instrument making. (Hilyard, Laopodis, Faber 1999) ${ }^{1}$ (Clemens, Crain, Anderson 2004) ${ }^{2}$ (Epps, Sutin 2003) ${ }^{3}$ (Sheinis et. al. 1999) $^{4}$ (Buckley et. al. 2008) ${ }^{5}$ (Brown, Fabricant, Boyd 2002) ${ }^{6}$

Fluid

Glycerin

Cargille Laser Liquid Code 1074

Cargille Laser Liquid Code 5610

Cargille Optical Gel Code 081160

\section{Material}

Delrin

Polyethylene

Polyurethane

Kapton

Teflon

Silicone
Instruments that Use Material

None

DEIMOS ${ }^{1}$, SDSS $^{2}$

IMACS $^{3}, \mathrm{ESI}^{4}$, UNC Goodman Camera ${ }^{5}$

$\mathrm{RSS}^{5}$, Binospec ${ }^{6}$

None

ESI

DEIMOS

None

None

RSS

$\mathrm{LRIS}^{5}$ and RSS 


\section{CHAPTER II}

\section{METHOD}

\section{Setup}

The entirety of our methodology revolves around measuring the absorption spectra of the index-matching fluid (henceforth referred to as the 'sample') as it becomes contaminated by the common telescope material. Our setup is simple but has many nuances that are necessary to make it work.

At its simplest, our setup consist of a light source, the sample, and a spectrometer. The light is provided by a xenon lamp which has an emission spectra that extends into the UV range as we require for this experiment. The sample is contained in a $100 \mathrm{~mm}$ path length, quartz cuvette. The spectrometer is calibrated properly so that it can detect any possible absorption in the UV.

The setup is complicated by the problem of optically coupling the lamp, the sample, and the spectrometer. The lamp generates light which is fed into the monochromator and consequently into the fiber that is coupled to it. We do not use any of the monochromator's features (i.e. we do not select any particular wavelength in our spectra). Instead, the monochromator is used purely as a means of optically coupling the lamp to the first optical fiber (we shall explain that later). 


\section{Optical coupling in setup}

We use a high-end fiber (FTO-100601) to transmit light from the monochromator to the cuvette holder. This fiber has such good transmission that it will not be the 'weak link' if too much extraneous UV absorption in our experiment setup. This high-transmittivity fiber is SMA-1 coupled to the cuvette holder. Near each side of the cuvette holder is a lens that channels the light from the fiber into parallel rays through the cuvette.

At this stage, the light will pass through quartz cuvette holding the sample. Quartz has high UV transmittance so once again we do not need to worry about it being a 'weak link'. So then, at this point the sample would absorb light based on its degree of UV contamination, and we would then be able to measure that.

But before we run our actual experiment, we need to optimize our setup for the equipment available to us. One thing we must optimize is the second fiber which is the most likely to be the 'weak link' in the setup because we do not have a second high-end fiber to use. Instead, we use a decent fiber, but several middle-grade fibers have significant UV absorption so we measured several fibers to find one that is acceptable for our experiment. The results of this test are presented in figure 3. We can see that each fiber transmits about the same so we picked QP200-2-UV-VIS because of its short length which usually has less attenuation anyways. 
UV Throughput Comparison of 5 Fibers

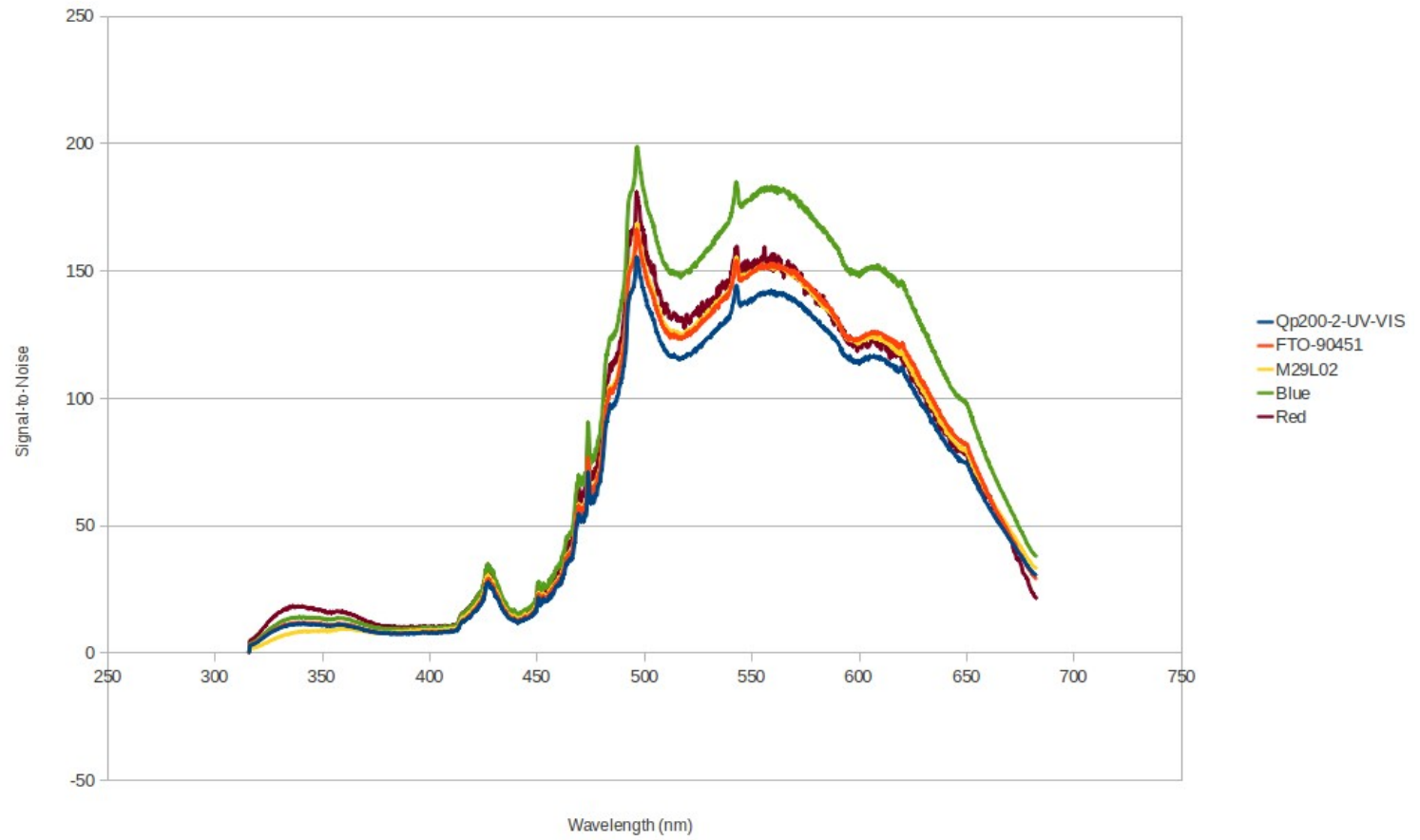

FIG. 3- UV Throughput Comparison of 5 Fibers. The throughput spectra of all the fibers is about the same so using any one of the fibers in our setup won't make much of a difference.

\section{Monochromator grating in setup}

To optimize the test further, we measure the setup's emission spectra for various gratings in the monochromator. We do this because a UV grating will diffuse light more than an IR grating, and thus a UV grating will attenuate the amount of UV light emitted which we do not want. The results of this test are presented in Figure 4. We can see that each grating seems to have two halves of its spectra: the low intensity UV half and the high intensity optical half. We decided to use $1200 \mathrm{~nm}$ grating because it has the flattest spectra over each of the two halves. 
4 Different Grating Blaze Angle's Signal-to-Noise

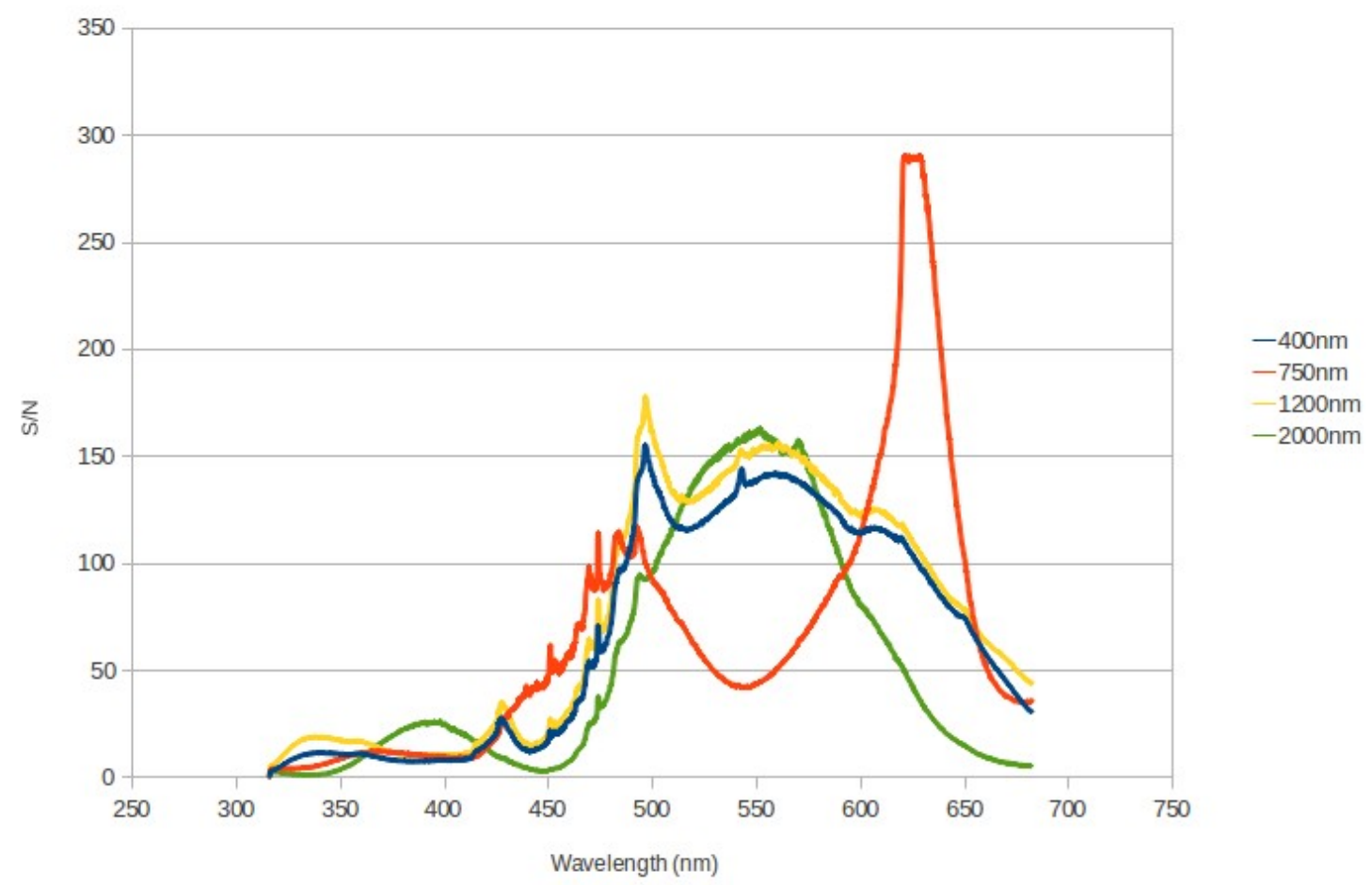

FIG. 4- 4 Different Grating Blaze Angle's Signal-to-Noise. The different grating's spectra have very different shapes. We want the $\mathrm{S} / \mathrm{N}$ to be above 100 for reduced error but below 250 to avoid nonlinear effects. Thus, flat-shaped spectra are better.

\section{Two phase measurement}

The flatness of the spectra can aid in maximizing the signal-to-noise without

illuminating the CCD into its nonlinear detection region. For example in Figure 4, the spectra for the 750nm grating has a flat peak at 650nm because the CCD was saturated in this region. Thus, that measurement is inaccurate because some photons were counted multiple times (nonlinear) and other photons were not counted at all (saturation). We could turn down the integration time of the CCD but that would lower the signal-tonoise for the rest of the spectra. Therefore, the signal-to-noise of the entire spectra is 
bounded by that of the highest peak in the spectra which makes spectral flatness a very crucial feature in a grating.

\section{Signal-to-Noise for 2 Phase Test}

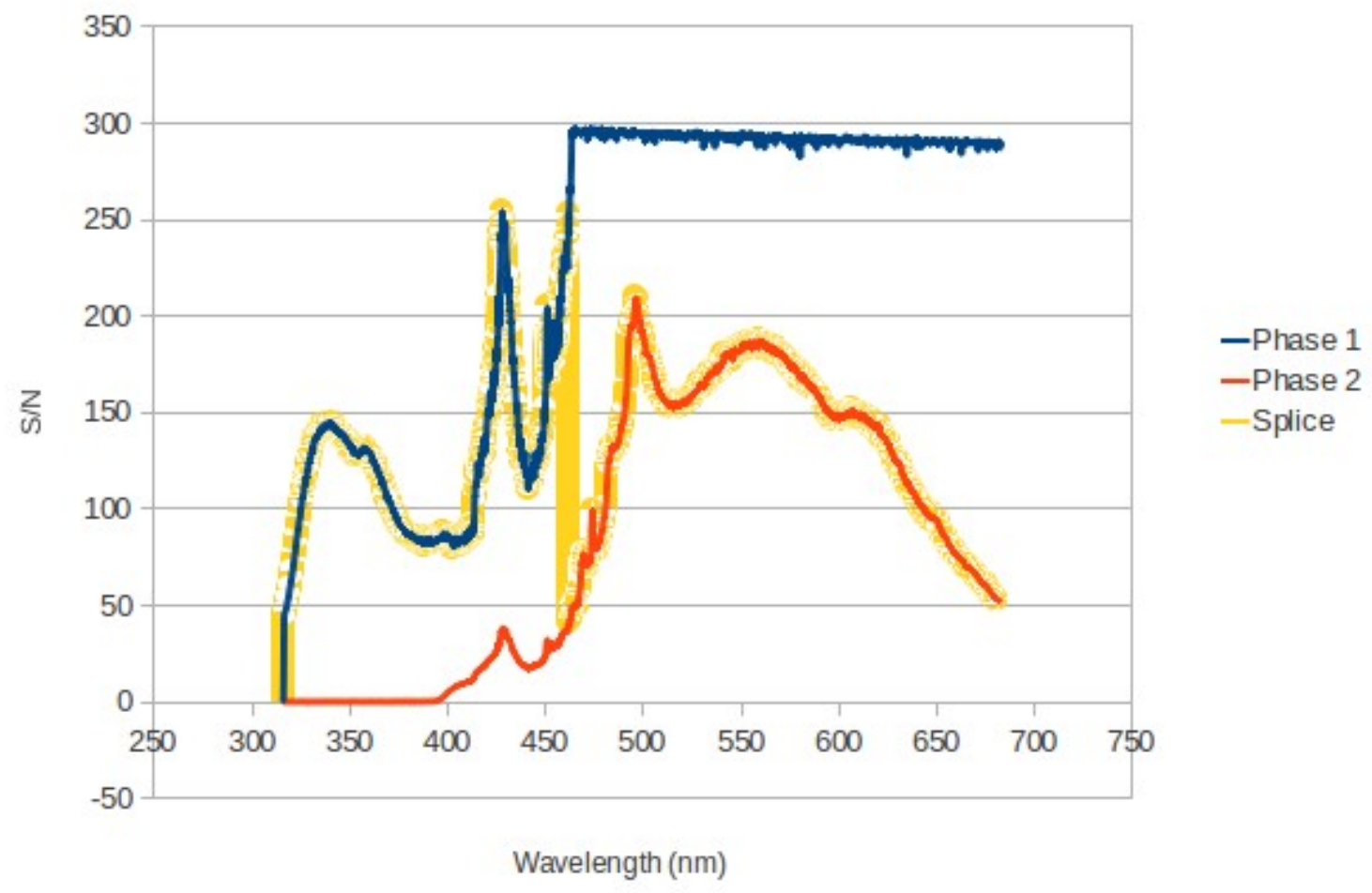

FIG. 5- Signal-to-Noise for 2 Phase Test. In this measurement, phase 1 has an integration time of 200ms, and phase 2 has an integration time of 30ms. Splicing the best parts of each measurment gives us a measurement with much better overall $\mathrm{S} / \mathrm{N}$.

The fact that each grating's spectra consists of two halves of different intensity can be corrected given that each half is spectrally flat. We simply divide the measurement into two measurements with two different integration times. The result of this two phase approach for the final setup is presented in Figure 5. The spliced measurement then has a 
signal-to-noise above 100 for the majority of the spectrum which reduces the measurement error introduced by noise to $1 \%$.

\section{Final Setup}

BOB Monochromator w/
Xenon Lamp and 1200nm Grating

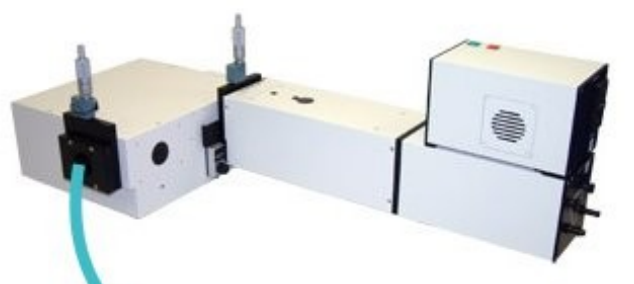

Fiber Tech Optics FTO-100601

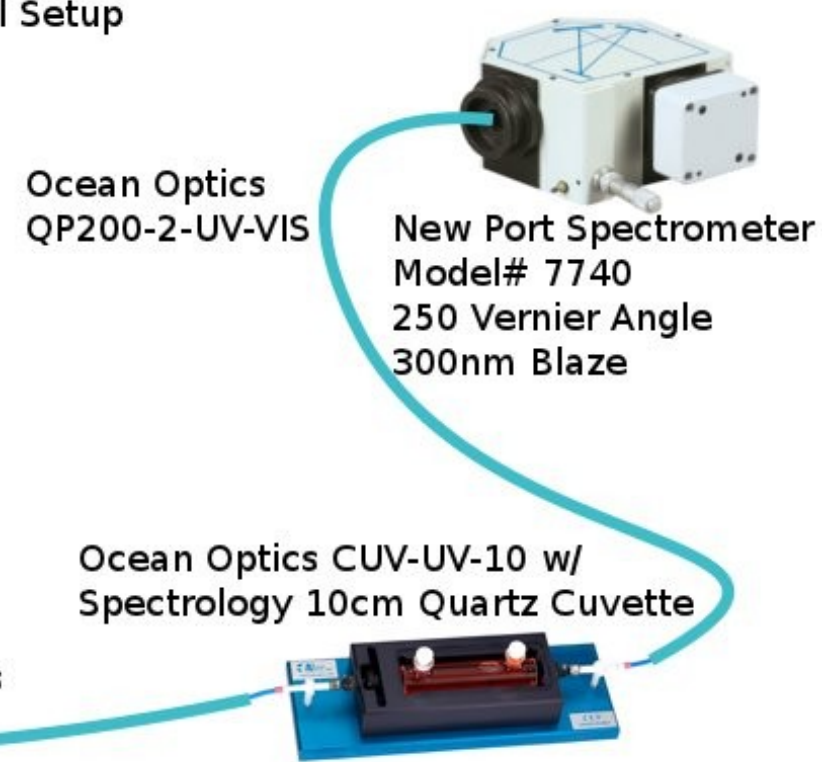

FIG. 6- Final Setup. Light generated by the lamp is coupled by the monochromator into the fiber. It then passes through the sample and into the second fiber and then the spectrometer where its spectra is measured.

\section{Final setup and procedure}

We have now maximized our setup (presented in Figure 6) and are ready to begin. To facilitate the repeatability of results, we have recorded a lab procedure that will guide the process of testing of fluid and material pairs. We list this procedure below.

How to setup contaminant for aging

1) Cut the contaminant into enough uniform pieces.

a) Cut one piece for each fluid being tested.

b) Make sure each piece has the same dimensions and same smoothness to each 
side because we need them to have the same active surface area.

2) Clean the contaminant and one flask with water.

a) It is important that the flask's cavity and the test contaminant be cleaned of extraneous contaminants.

3) Clean equipment from step 2 with acetone.

4) Let acetone evaporate.

a) It would be best to apply heat to cuvette.

5) Place a flask on a scale and zero out scale

6) Pour fluid directly from product container into flask

a) Target volume is $40 \mathrm{~mL}$

b) Underfill cuvette, weigh fluid, and calculate volume using fluids density from data sheet.

c) Add more fluid and repeat substep $6 \mathrm{~b}$ as necessary.

d) This volume should be as exact as possible to preserve active surface area between tests.

7) Slowly place one sample into the flask.

8) Immediately start sample timer.

9) Repeat steps 2 through 8 for the other fluids for that contaminants.

10) Repeat steps 1 through 9 for the other contaminants. 
How to measure spectra of contaminated fluid

1) Make sure fibers of the cuvette holder are collimated properly.

1) Do not move the fibers during measurements. The contaminated and control measurements need to have the same optical setup.

2) Turn on the xenon lamp light source.

1) Its spectrum must be stable before measurements.

3) Clean two cuvettes with water and then acetone as described earlier.

4) Fill one cuvette with $25 \mathrm{~mL}$ of clean index-matching fluid for the material and fluid pair that is being test.

1) Use the technique described in step 6 of the setup procedure

2) This volume should be as exact as possible so that the path length is not filled with air.

3) Cap the cuvette and make sure the sample does not contact the cap.

5) Place the clean sample cuvette into the cuvette holder and close the casing.

6) Measure the absorption spectrum of the clean sample.

7) Remove the cuvette and pour the clean fluid back into its container.

8) Fill the other cuvette with $25 \mathrm{~mL}$ of contaminated index-matching fluid for the material and fluid pair that is being tested.

1) Use the technique described in step 6 of the setup procedure.

2) This volume should be as exact as possible to preserve active surface area between tests and to prevent air from filling the path length. 
3) Cap the cuvette after filling and make sure the sample does not contact the cap.

9) Place the contaminated sample cuvette into the cuvette holder and close casing.

10) Measure the absorption spectrum of the contaminated sample.

11) Remove the cuvette and pour the sample back into its container.

12) Measure its absorption spectrum.

13) Return both containers (clean and contaminated) back to storage.

1) Make sure to place the cap securely on the flask.

2) Make sure that the sample does not contact the cap while in storage. 


\section{CHAPTER III}

\section{RESULTS}

\section{Preliminary test}

Performing the full experiment necessarily does irreversible damage to the expensive

fluids we seek to test. So, we performed a preliminary test with glycerin and delrin which is the least expensive fluid/material pair. The results of this trial are depicted in Figure 7.

\section{Glycerin w/Delrin: Transmission Curves after Contamination}

Decrease due to water absorption/index gradient

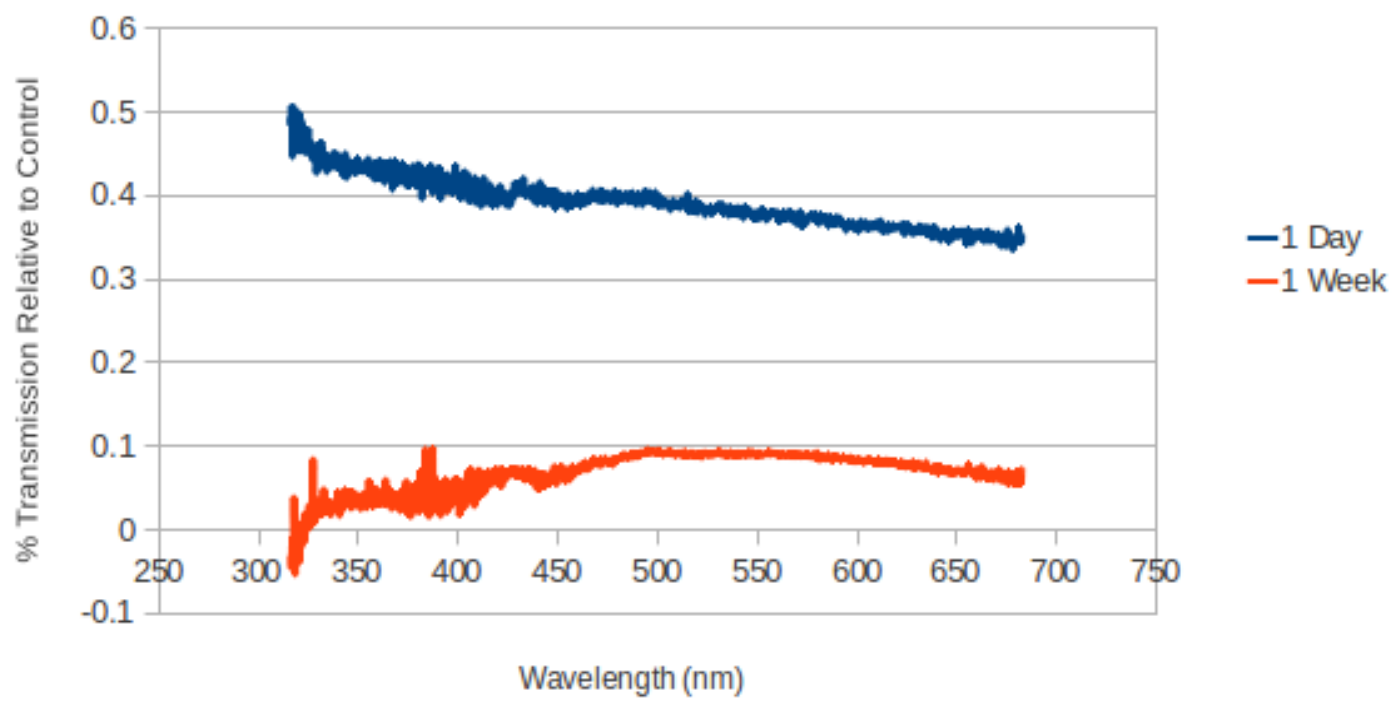

FIG. 7- Glycerin w/Delrin: Transmission Curves after Contamination. We made a measurement of the sample's spectra before contamination on the zeroth day and used this as the baseline. The percent transmission for one day and one week of aging are much smaller than expected because of systematic errors. 
Fortunately, several systematic errors of our setup were evident in this test. We say this is fortunate because these errors were both easy to spot and easy to fix. We adjusted our experiment procedure to rectify these errors in our final experiment.

The first systematic error causes the one day measurement to attenuate unnaturally quickly. This error was the day-to-day variations in the intensity generated by our setup. This can be produced by misalignments in the optical coupling, variability in the intensity of the xenon lamp, or many more reasons. The problem is that we are using the zeroth day measurement as the control when in fact we should be measuring an uncontaminated sample and using that as the control for each day individually.

The second systematic error caused the one week measurement to drop to near zero. Upon examining the setup, we discovered that light path in the cuvette was being refracted to the extent of breaking the optical coupling. So we removed the cuvette and observed that this effect persisted regardless of the cuvette orientation. Also, we stirred the glycerin and observed that the effect disappeared. It is well known that glycerin is hygroscopic so these few simple test allowed us to deduced that the glycerin had absorbed water from the air which produced a gradient in the refractive index of the sample. This gradient deflected the light from its normal path. The solution to this is to keep the sample in a closed container at all times. 


\section{Aging the contaminated samples}

With the knowledge that each sample must be isolated from air, we will fill 24

volumetric flasks each with a different combination of the four fluids and six materials.

These pairs will age, and the results of this will be released at a later date. 


\section{CHAPTER IV}

\section{SUMMARY AND CONCLUSIONS}

\section{Summary}

In this paper, we have described a preview of our experiment which will test the compatibility of several index-matching fluids with several common astronomical instrumentation materials. We will be using our results to prescreen fluid and material pairs for the GMACS spectrometer but we have also shown that the fluids and materials that we are testing are currently being used by many popular instruments. We have devised a simple setup to measure the rate of degradation of UV transmission for the samples. We optimized this setup by performing test to find the best fiber and grating available at the time of the experiment. We improve the overall signal-to-noise of our setup by performing two separate measurements of our absorption spectrum (from 320460nm and from 460-700nm) with two separate integration times (200ms and 30ms respectively). Our practice test using the glycerin and delrin pair revealed two systematic errors: day-to-day variability of the setup and air contaminating the sample. We corrected this by including a day-to-day control measurement in the procedure and by keeping caps on both the storage flask and testing cuvettes. The experiment setup is ready to begin testing, and the samples are ready to begin aging. 


\section{Conclusions}

The RSS team found that their UV transmission dropped to $10 \%$ of its original transmission for a path length of 1mm over a time frame of about 2 years with polyurethane as the material and LL5610 as the optical fluid. Because the transmission drops exponentially for both path length and time (i.e. if we doubled either the path length or time, the transmission would drop by $10 \%$ again to now $1 \%$ of its original transmission), we can use this to calculate the $10 \%$ transmission time for our experiment's path length of 100mm with the polyurethane and LL5610 sample. We find that this time is about one week. Therefore, we could easily affirm or deny the presence of contamination after aging the samples for one month which has equivalent transmission loss to that of a $1 \mathrm{~mm}$ path length sample aged for four years.

We designed this experiment with the intentions of having the complete results available for the 2012 SPIE Instrumentation Summer Conference in Amsterdam on July 1, 2012. Considering that we have the experiment setup completed as of the writing, we expect to still be on track for that goal because it should take only a month to age the samples which leaves another two months for data analysis and any unexpected delays. 


\section{REFERENCES}

Brown W. R., Fabricant D. G., Boyd D. A., 2002, PASP, 114

Buckley D. A., Barnes S. I., Burgh E. B., Crawford S., Cottrell P. L., Kniazev A., Nordsieck K. H., O'Donoghue D., Rangwala N., Zanmar Sanchez R., Sharples R. M., Sheinis A. I., Vainsanen P., Williams T. B., 2008, Proc. SPIE, 7014, 6

Clemens J. C., Crain J. A., Anderson R., 2004, Proc. SPIE, 5492, 331

Epps H. W., Sutin B. M., 2003 , Proc. SPIE, 4841, 612

Hilyard D. F., Laopodis G. K., Faber S. M., 1999, Proc. SPIE, 3786, 482

Lloyd-Hart M., Angel R., Milton N. M., Rademacher M., Codona J., 2006, Proc. SPIE, 6272

Marshall J. L., DePoy D. L., Shectman S. A., Papovich C., Smee S. A., Barkhouser R. H., Prochaska T. M., Hammond R. P., 2012, AAS Meeting \#218, \#422.13

Nordsieck K., Nosan F., Schier A. J., 2010, Proc. SPIE, 7735, 262

Sheinis A. I., Sutin B., Epps H., Schier J. A., Hilyard D., Lewis J., 1999, Proc. SPIE, 3786, 413 


\section{CONTACT INFORMATION}

Name:

Professional Address:

Email Address:

Education:
Tyler William Behm

c/o Dr. Darren DePoy

Department of Physics and Astronomy

TAMU 4242

Texas A\&M University

College Station, TX 77843

tylerwbehm@gmail.com

B.S., Physics and Math, Texas A\&M University, Dec. '12 Undergraduate Research Scholar

Goldwater Scholar

Astronaut Scholar

3 Years at TAMU Astronomical Instrumentation Lab SAO/Harvard Solar REU

NSO Solar REU 